\title{
Some Implications of the Anisotropic Distribution of Satellite Galaxies
}

\author{
Dennis Zaritsky ${ }^{1}$ and Anthony H. Gonzalez \\ UCO/Lick Observatories and Board of Astronomy and Astrophysics, \\ Univ. of California at Santa Cruz, Santa Cruz, CA, 95064 \\ dzaritsky@as.arizona.edu, anthonyg@ucolick.org
}

\begin{abstract}
We examine a possible connection between the anisotropic distribution of satellite galaxies around giant spiral galaxies and the evolution of satellite systems. The observed polar anisotropy (Zaritsky et al. 1997) is either imprinted by initial conditions or develops from an initially symmetric distribution. We attempt to discriminate between these two possibilities by exploring the implications of the latter one. From the observed distribution of satellite galaxies relative to the primary galaxy's disk, we derive constrains on the orbital inclinations of the current satellite population. Using this derived inclination limit and assuming that the initial population had no preferred orbital inclination, we estimate the size of the hypothesized original population. We find that our best-fit models imply a population of destroyed (or inhibited) satellites whose combined luminosity (assuming the same $M / L$ as for the observed satellites) is between $18 \%$ and $103 \%$ of the current disk luminosity.
\end{abstract}

Subject headings: galaxies: formation — galaxies: interactions

\section{Introduction}

Disk galaxies presumably form from protogalactic clouds that consist of at least several sub-galaxy aggregates, some fraction of which eventually merge to form the dominant galaxy. How efficient and complete is this hierarchical process? Quantitative answers to this type of question have been solely in the domain of simulations (cf. Navarro and Steinmetz 1997 ; Klypin et al. 1999 (hereafter K99); Moore et al. 1999 (hereafter M99)). Those simulations are in turn constrained by observations of the properties of present day galaxies (such as the local Tully-Fisher relation), which are the result of a variety of complicated physical processes, and of the properties of high redshift galaxies, which are difficult to quantify and influenced by selection biases. A reasonable goal is to find a more direct link to the process of hierarchical formation.

\footnotetext{
${ }^{1}$ Current address: Steward Observatory, Univ. of Arizona, Tucson, AZ, 85721
}

In hierarchical formation scenarios, small mass objects generally collapse prior to large ones and become the building blocks of larger objects. Two types of objects that currently surround giant galaxies may qualify as possible hierarchical building blocks: globular clusters and satellite galaxies. Most studies of the dynamical evolution of either globular clusters or satellite galaxies begin with an assumed initial distribution of such companions and focus on the subsequent dynamical evolution of the system. For example, Aguilar, Hut, \& Ostriker (1988) calculate the rate of destruction of globular clusters to assess whether the galactic spheroid was built from globular clusters, and Ostriker \& Tremaine (1975) investigate the luminosity evolution of the primary galaxy due to infalling satellites. A difficulty with this approach is that the results depend sensitively on the unknown characteristics of the initial population and that we have no empirical means of determining whether the observed population represents a small or large fraction of the initial population. 
High-resolution numerical simulations are beginning to produce populations of low-mass companions around giant galaxies in a self-consistent cosmological framework (K99 and M99), but they produce far more satellite galaxies than observed.

The observed distribution of satellite galaxies of spiral primaries out to $500 \mathrm{kpc}$ is asymmetric and elongated along the disk minor axis (Odewahn 1989; Zaritsky et al. 1997, hereafter ZSFW, and $H_{0}=75 \mathrm{~km} / \mathrm{s} / \mathrm{Mpc}$ assumed throughout). This elongation is an extension of the Holmberg effect (the preferred polar orientation of satellites interior to $r \sim 50 \mathrm{kpc}$; Holmberg 1969) to larger radii - although the physical causes of the two observational results may differ and the polar elongation is not evident at intermediate radii (50 to 200 $\mathrm{kpc}$ ). Both the inner and outer satellite results are statistical because orbits of individual satellites are unknown (although the ZSFW result is for kinematically confirmed satellite galaxies). In the one galaxy for which individual satellite orbits are known, the Milky Way, there is evidence that the orbits are preferentially polar from the alignment of satellites on the sky (Kunkel \& Demers 1976; Lynden-Bell 1982), the orientation of the Magellanic Stream (Mathewson, Cleary, and Murray 1974), the three-dimensional distribution of satellites (Majewski 1994; Hartwick 1996), and their space velocities (Scholz \& Irwin 1994). Finally, Grebel, Kolatt, \& Brandner (1999) find tentative evidence for a statistical excess of M 31 satellites along a polar orbit. Hence, preferentially polar satellite orbits may be common.

The connection between disks and satellite orbits is either imprinted in the initial conditions or is the result of dynamical phenomena during the formation and subsequent evolution of the galaxy. ZSFW argue that the orbital decay time due to dynamical friction at radii larger than $200 \mathrm{kpc}$ excludes dynamical friction as the dominant mechanism. For example, models of the effect of dynamical friction on the orbit of the Large Magellanic Cloud (Tremaine 1976) suggest that the perigalacticon distance has decreased by a factor of 3 in 10 Gyr. Therefore, satellites similar to the Large Magellanic Cloud that began at perigalacticon radii $>200 \mathrm{kpc}$ will not have merged with their parent galaxies. Quinn \& Goodman (1986), in their investigation of the Holmberg effect, found that dynamical friction could not even account for the asymmetry inside $50 \mathrm{kpc}$.

If we wish to invoke a dynamical process for the origin of the anisotropy, we must hypothesize that the missing satellites experienced a catastrophic event that either destroyed the satellite or inhibited the formation of stars (such as the removal of gas from the proto-satellite). Such an event is most likely to occur as satellites make a pericentric pass near the giant, and so could only affect satellites that have made at least one pericenter passage. Using the standard orbital equations of the timing argument (Kahn \& Woltjer 1959), a halo mass of $1.5 \times 10^{12} M_{\odot}$ (the $90 \%$ confidence lower mass limit for the mass enclosed at $200 \mathrm{kpc}$ for this sample of primary galaxies: Zaritsky \& White 1994), and $t_{0}=15$ Gyr for the age of the Universe, we find that any satellite on a radial orbit at a current distance $<530 \mathrm{kpc}$ has made at least one pericenter passage. If (1) satellite orbits are highly radial (as found in recent simulations: K99 and M99) and (2) satellites on planar orbits either preferentially lose a greater amount of orbital energy, have their gas removed, or are disrupted near pericenter, then anisotropy in the satellite population might extend to radii as large as $500 \mathrm{kpc}$. The currently available simulations (K99 and M99) do not show such satellite destruction, but these models do not include gas and the survivability of satellites is sensitive to the particulars of the simulation, such as details of the power spectrum (M99). Whether satellite destruction is more common for satellites on planar orbit and whether the mechanism is sufficiently severe to induce the asymmetry has not been demonstrated and must be investigated further. To continue our exploration of the evolution hypothesis, we postulate that such a mechanism does exist and follow the argument to its conclusion.

Regardless of the exact dynamical model that may lead to the anisotropy in the evolution conjecture, we can use the anisotropy to estimate the toll that the process has exacted on the satellite population. We do this by (1) constraining the range of satellite orbital inclinations allowed by the ZSFW sample and (2) estimating the size of the initial satellite population by assuming that it was initially spherically symmetric. Is the inferred missing population a significant component of the galaxies or are the results so implausible that they enable us to exclude the evolution conjecture? The 
methods used to constrain the orbital inclinations are discussed in $\S 2$. After determining the number of "missing" satellites, we assess whether the destroyed satellites constitute a significant fraction of the mass of the primary galaxy. The results and implications are discussed in $\S 3$.

\section{Determining the Orbital Inclination Limit}

Because projection effects and the wide range of viewing angles partially mask any underlying asymmetric satellite distribution, an observed asymmetric distribution implies a more strongly asymmetric underlying distribution. To determine the degree of polar alignment necessary to reproduce the observed distribution, we determine the orbital inclination limit in three different ways. In all three ways we presume that there is a single lower inclination limit for satellite orbits.

Our first approach is adopted from Quinn and Goodman's (1986) treatment. For assumed circular orbits and a power-law radial density profile (parameterized by $\rho \propto R^{\beta}$ ), they derive an analytic expression for the surface density as a function of angle from the disk plane. In Figure 1 , we plot the number of satellites as a function of angle from the plane, $\theta$, and compare the results from Quinn and Goodman's calculation for an orbital inclination limit of $45^{\circ}$ and $\beta=1.8$ (as measured for satellite galaxies : Lake \& Tremaine 1980, Zaritsky et al. 1993, Lorrimer et al. 1994)). For comparison, we plot the number of satellites vs. $\theta$ for satellites at $r>200 \mathrm{kpc}$ (for which the anisotropy appears stronger). This comparison suggests that an orbital inclination limit of $45^{\circ}$ (we define the inclination limit to be measured from the pole) is appropriate for the full sample and that this limit is tighter for the outer satellites.

Quinn \& Goodwin's calculation is independent of the assumption of circular orbits, as long as the orbits are not closed and one time averages as the apsides of eccentric orbits precess. However, the satellites at large radii in our sample have not completed many orbits, the apsides have not precessed, and so this assumption may be inadequate. A second possible shortcoming of the calculation is that interlopers, apparent satellites that are not physically associated with the sys- tem, are not included. The estimated fraction of interlopers for this sample is between 10 and $15 \%$ (Zaritsky 1992).

We proceed by examining models with Keplerian orbits that include interlopers. The satellite orbits are taken from a family of fixed eccentricity orbits for any single model (although we explore a range of eccentricity values across all models). The orbital energy is drawn from the power-law distribution given by Bahcall and Tremaine (1981)

$$
P(E)= \begin{cases}(3-s) E^{s-4} / E_{0}^{s-3}, & \text { if } E>E_{0} \\ 0, & \text { if } E \leq E_{0},\end{cases}
$$

where $s<3$ and $E=(G M / r)-\left(v^{2} / 2\right)$. This choice of $P(E)$ generates a number density profile of test particles that is proportional to $r^{-s}$ for $E>E_{0}$. We choose $s=1.8$ and $E_{0}=0.0065$ to match the observed mean projected separation, $\left\langle r_{p}\right\rangle(\sim 200 \mathrm{kpc})$, and the radial number density profile (Lake \& Tremaine 1980; Zaritsky et al. 1993; Lorrimer et al. 1994). The mass ratio between the satellite and primary is chosen to be 1:20 (comparable to the mean observed ratio assuming equal M/L's for primary and satellite which is $\sim 1: 13$ ). The mean anomaly (orbital phase) is selected uniformly from $(0,2 \pi]$. The satellite orbits are then randomly oriented using the Euler angle convention and the known distribution of primary disk inclinations for the ZSFW primaries. Simulated orbits are accepted only if the angle between the orbital major axis and the primary disk's rotation axis is $\leq \theta_{l}$, where $\theta_{l}$ is the orbital inclination limit. In exploring the models we vary $s$, $e, \theta_{l}$, and $f_{I N T}$, where $f_{I N T}$ is the fraction of the sample that consists of interlopers. From observations, we limit $s$ to between 1.5 and 2 (Lorrimer et al. 1994). On the basis of ZW's infall simulations we limit $e$ to between 0.5 and 0.9 , with a preferred value of 0.7 . Finally, from various arguments (Zaritsky 1992), our preferred value of $f_{I N T}$ is 0.1 , but we also explore $f_{I N T}=0.05$ and 0.15 . For each model, we generate 10,000 artificial satellites.

We compare the results of these simulations to four subsamples of the ZSFW satellite galaxies. Sample 1 includes all of the satellites of all of the primaries in the ZSFW sample. Sample 2 includes only those satellites beyond $r_{p}=300 \mathrm{kpc}$, and so is limited to the radial range where a strong azimuthal asymmetry is evident (Figure 2). Sample 
3 includes all of the satellites of the primaries with disk inclination angles $>45^{\circ}$. This sample is less affected by projection and confusion between polar orbits and those in the disk plane. Sample 4 includes only those satellites beyond $r_{p}=200 \mathrm{kpc}$ that are associated with primaries with disk inclination angles $>45^{\circ}$. The inner radial limit for Sample 4 is decreased from 300 to $200 \mathrm{kpc}$ relative to Sample 2 because the asymmetry is evident in this sample down to $r_{P} \sim 200 \mathrm{kpc}$ and the number of satellites beyond $r_{P}=300 \mathrm{kpc}$ is smaller (this information is summarized in Table 1). To compare the simulations with the data, we calculate the two-sided KS statistic ${ }^{2}$ for the distributions of satellite position angles relative to the disk major axis. The results for the best fit $\theta_{l}$ and $90 \%$ confidence interval are presented for various models and data samples in Column (6) in Table 2. Again, we conclude that the most likely value of the orbital inclination limit for the full sample lies around $45^{\circ}$ and that this limit for the outer sample is smaller (about $20^{\circ}$ ). However, the uncertainties on the derived $\theta_{l}$ are large.

As discussed by Zaritsky and White (1994), the full description of the dynamics of satellite systems requires a model of the growth of the primary galaxy's halo with time and the evolution of the satellite population within that halo. In particular, the assumption that satellites are currently found at a random phase along an orbit (which is necessary for the Keplerian models) is suspect for satellites at large radii, where the orbital period is $\sim$ Hubble time. Therefore, we proceed to test the results from the Keplerian models (for which parameter space is easily explored) with the results of the spherical-infall halo simulations used by Zaritsky \& White (1994) to measure the mass of galaxy halos. Using the simulation that best matches their best fit parameters $\left(\Omega_{0}=0.3\right)$, we have derived preferred orbital inclination limits for the four satellite subsets. We present those results in Column (7) of Table 2. The best fit values are indistinguishable from those derived using the Keplerian models, but the $90 \%$ confidence ranges vary. In particular, we find that the entire range of $\theta_{l}$ is allowed when the satellite sample includes satellites at all radii, and that none of the range

\footnotetext{
${ }^{2}$ This statistic is better suited to the analysis of data with no natural minimum or maximum (as is the case for position angles) than the standard KS test (Press et al. 1992).
}

is within the $90 \%$ confidence limit when only the outer satellites are considered (indicated by [-, ]).

We conclude that all three analysis techniques indicate similar best fit limits $\left(\sim 15^{\circ}\right.$ to $\left.60^{\circ}\right)$, but that strong (e.g., $90 \%$ confidence) statistical conclusions cannot yet be reached. Because of the agreement among the various methods used to determine $\theta_{l}$, the current principal limitation does not appear to lie in the details of the models, but rather with the sample size.

\section{Discussion}

For all samples and all model parameters within our specified ranges, the best fit values of $\theta_{l}$ indicate that the orbits are preferentially polar. The best fit $\theta_{l}$ for Sample 1 and our reference model $\left(s=1.8, e=0.7\right.$, and $\left.f_{I N T}=0.1\right)$ indicates that all of the satellites are on orbits that are inclined at least $38^{\circ}$ to the disk plane $\left(\theta_{l}=52^{\circ}\right)$. Over the range of radii where the asymmetry is most pronounced $(>200 \mathrm{kpc}$ ) for systems with primaries highly inclined to the line-of-sight $\left(>45^{\circ}\right)$, the best-fit solutions indicate that the orbits are confined to within $20^{\circ}$ of the pole (for either Keplerian or Infall models) and that the orbits are confined to within $\sim 60^{\circ}$ of the pole with greater than $90 \%$ confidence.

Before drawing conclusions from these results, we discuss the sensitivity of the models to various parameters. Our reference model is defined to have $e=0.7, s=1.8$, and $f_{I N T}=0.1$ (the results from this model, as applied to Sample 4, are presented as Model 2 in Table 2). We test all of these choices with the Keplerian models. First, we vary $e$ between 0.5 and 0.9 (the $90 \%$ confidence limits derived by ZW; Models 1 and 3 ). This parameter sometimes has a noticeable effect on $\theta_{l}$, so we present results for all three eccentricities. Second, we vary $s$ between 1.5 and 2.0 (Models 4 through 9 in Table 2). The results are nearly insensitive to $s$. Third, we vary the interloper fraction between 5 and $15 \%$ (Models 10 through 15). As with $s$, changing this parameter has a minimal effect on $\theta_{l}$. We also present results derived using other subsamples (for the standard parameter choices and $0.5 \leq e \leq 0.9$; Models 16 through 24)

We now determine the number of "missing" satellites implied by a particular $\theta_{l}$. From the 
range of allowed orbital inclinations, we calculate the fraction of all allowed orbits that are represented in the current sample. This fraction is equivalent to the fraction of the volume of a sphere that lies within $\theta_{l}$ of the pole, which equals $1-\cos \theta_{l}$. For an opening angle of $30^{\circ}$, the volume within the allowed cone is $13.4 \%$ of that within the sphere. If the "original" population of satellite orbits uniformly filled the sphere and our value for $\theta_{l}$ is $30^{\circ}$, then the current population is only $13.4 \%$ of the original population, or originally there were 7.5 times as many satellites as there are in the observed sample.

The interpretation of $\theta_{l}$ and the "missing" satellite population is complicated by the apparent change in the magnitude of the polar asymmetry at different radii. The data in Figure 2 suggest that in addition to the asymmetry at $r_{P}>300$ kpc, there may be a slight excess of planar satellites at $\sim 180 \mathrm{kpc}$ and a slight excess of polar satellites once again at small radii. Although our simulations illustrate that projection effects result in less apparent asymmetry at small radii even in a model where the limit on orbital inclination is the same at all radii (see Figure 2), we do not reproduce the observed dip in polar angle at $\sim 180 \mathrm{kpc}$. However, this apparent disagreement is not statistically significant ( significance $\sim 1 \sigma$ ) because of the small number of satellites in each radial bin. The gradual radial increase in the number of satellites $\left(\propto r^{0.2}\right)$ and the lack of a strongly planar asymmetry between 0 and $200 \mathrm{kpc}$ suggest that a large number of outer, planar satellites cannot be hidden as satellites at smaller radii, unless there is a destruction of a comparable number of inner planar satellites to compensate. Therefore, we can calculate the number of "missing" outer satellites and use that quantity as an estimate of the total number of "missing" satellites.

The dependence of the polar asymmetry on radius leads to the result that even an orbital inclination limit of $90^{\circ}$ is allowed within the $90 \%$ confidence limit when satellites at all radii are included (Samples 1 and 3). The lack of a strong polar signature in the complete sample weakens the claim of polar alignment and argues for larger samples to resolve the issue. However, we remind the reader that the polar alignment at large radius is highly significant (ZSFW) and that polar alignements have also been observed both in the satellite system of our galaxy (cf. Hartwick 1996) and in the Magellanic Irr satellites of other galaxies (Odewahn 1989).

We now estimate the number of satellites destroyed, inhibited, or accreted by field giant spiral galaxies. If $\theta_{l}=18^{\circ}$ (best fit for Keplerian Model 2) for the outer satellite population, a large population of corresponding disk plane satellites (19 times the current number of satellites beyond $\left.r_{p}=200 \mathrm{kpc}\right)$ are missing. For the average primary galaxy in our sample, this estimate implies a loss of 13.4 satellites of comparable luminosity as the satellites in our sample (for the $i>45^{\circ}$ sample). However, due to the small sample size we cannot exclude the possibility with greater than $90 \%$ confidence that the missing population is only comparable in size to the current population (0.7 satellites/primary at $r_{p}>200 \mathrm{kpc}$ ). The latter conclusion obviously places less stringent constraints on the possible current status of the missing satellites.

Over the suite of models and samples, we infer a wide range in the number of missing satellites. If we adopt our best fits for $\theta_{l}$ across all samples for our reference model, then the number of missing satellites inferred per galaxy ranges from 2.4 to 13.4. These values for the population of missing satellites are consistent with the number of "extra" satellites (with velocity dispersion $\gtrsim 30 \mathrm{~km} \mathrm{sec}^{-1}$ ) present in recent simulations (K99 and M99). The average luminosity of a primary in our sample is 13 times the average luminosity of a satellite (this calculation includes a completeness correction factor of 1.4 due to satellites that may have been missed in our spectroscopic survey as derived assuming a Schechter luminosity function with a faint-end slope $\alpha=-1.5$ ). Therefore, if the primaries have indeed accreted between 2.4 and 13.4 satellites, a significant fraction of their luminosity (18 to 103\%), and possibly their mass if the M/L's are comparable, comes from these satellites.

The best fit $\theta_{l}$ value for all of the satellites of all the primaries $\left(52^{\circ}\right)$ implies that we are missing 2.7 satellites per primary (or about $20 \%$ of the disk luminosity). If we assume that these satellites have been accreted, we can compare this value to the estimates of the satellite accretion rate from other studies. An extrapolation of the local accretion rate (Zaritsky \& Rix 1997) predicts that 
1 to 3 large satellites ( 7 to $21 \%$ of the current luminosity) are accreted over the lifetime of the galaxy. The actual number of satellites accreted over the lifetime of the galaxy is likely to be larger than the extrapolation of this estimate because the interaction rate is expected to increase with redshift. Within the large uncertainties in both approaches, the inferred satellite accretion rates are consistent and imply that satellite material may contribute significantly to the luminosity of the central galaxy. Interestingly, our investigation did not lead to predictions of satellite populations that had $L_{\text {Total }} \gg L_{D i s k}$, which would be implausible, or that had $L_{\text {Total }} \ll L_{\text {Disk }}$, which would have made this discussion academic.

We conclude that the evolution conjecture for the polar asymmetry has the following intriguing implications: (1) it enables an estimate of the size of the original satellite population, (2) the inferred population of "missing" satellites would have a luminosity of order that of the disk, and (3) the inferred number of missing satellites is consistent with the excess number of satellites produced by the most recent numerical simulations of galaxy formation (for satellites with velocity dispersions $\left.\gtrsim 30 \mathrm{~km} \mathrm{sec}{ }^{-1}\right)$. The principal difficulty with the evolution conjecture remains the unidentified physical mechanism necessary to destroy, remove, or inhibit, satellites on planar orbits with large apocenters.

\section{Summary}

We are searching for a signature of hierarchical galaxy formation in the properties of current spiral galaxies and their satellites. Satellites at large radii, or at least the components that would have formed those satellites, appear to have been preferentially "removed" from low inclination orbits (those in the disk plane) leading to the current preferentially polar distribution of satellites (ZSFW). Our quantitative estimate of the orbital inclination limit for the current satellite population has a large uncertainty - but, the best fit models imply that satellites on orbits within $70^{\circ}$ to $80^{\circ}$ from the disk plane at projected radii $>$ $200 \mathrm{kpc}$ have been destroyed, accreted, removed, or inhibited. We use these limits on the inclination of surviving orbits to estimate the number of "missing" satellites in low inclination orbits. The lost luminosity (or mass for constant M/L among satellites and primaries) is consistent both with an extrapolation of the local accretion rate and with the hypothesis that these "missing" satellites contributed substantial luminosity ( $\gtrsim 20 \%$ ) to the central galaxy. The large statistical uncertainties preclude us from determining whether the material in the "missing" disk plane satellites makes a modest $(\sim 10 \%)$ or dominant $(>50 \%)$ contribution to the luminosity and mass of the central galaxy. The identification of a lost satellite population may also help reconcile recent numerical simulations (K99, M99) that produce many more satellites per primary than observed. The principal weakness of this entire discussion is that no mechanism is demonstrated to appropriately affect satellites with large apocenter and low orbital inclination relative to the primary disk. The distribution of satellite galaxies provides a tool that, with more sophisticated simulations and larger samples, may enable us to further develop our understanding of galaxy formation and the dynamical evolution of galactic halos.

DZ acknowledges partial financial support from an NSF grant (AST-9619576), a NASA LTSA grant (NAG-5-3501), a David and Lucile Packard Foundation Fellowship, and a Sloan Fellowship. AHG acknowledges support from an NSF Graduate Student Fellowship. We thank A. Zabludoff for comments on a preliminary draft. 


\section{REFERENCES}

Aguilar, L., Hut, P., \& Ostriker, J.P. 1988, ApJ, 335,720

Bahcall, J.N., \& Tremaine, S. 1981, ApJ, 244, 805

Grebel, E. K., Kolatt, Ts., Brandner, W. 1999, in The Stellar Content of the Local Group, IAU 192, (eds.) P. Whitelock \& R. Cannon, (San Francisco: ASP Conf. Ser.), in press

Hartwick, F.D.A. 1996, in ASP Conf. Ser. 92, Formation of the Galactic Halo... Inside and Out, ed. H. Morrison \& A. Sarajedini (San Francisco: ASP), 444

Holmberg, E. 1969, Ark. Astron., 5, 305

Kahn, F.D., and Woltjer, L. 1959, ApJ, 130, 705

Klypin, A.A., Kravtsov, A.V., Valenzuela, O., \& Prada, F. 1999, ApJ, in press (astroph/9901240) (K99)

Kunkel, W.E., \& Demers, S. 1976, Roy. Green. Obs. Bull., 182, 241

Lake, G., \& Tremaine, S. 1980, ApJ, 238, L13

Lorrimer, S.J., Frenk, C.S., Smith, R.M., White, S.D.M., \& Zaritsky, D. 1994, MNRAS, 269, 696

Lynden-Bell, D. 1982, Observatory, 102, 202

Majewski, S. R. 1994, ApJ, 431, L17

Mathewson, D.S., Cleary, M.N., \& Murray, J.D. 1974, ApJ, 190, 291

Moore, B., Ghigna, S., Governato, F., Lake, G., Quinn, T., Stadel, J., \& Tozzi, P. 1999, submitted (M99)

Navarro, J.F. \& Steinmetz, M. 1997, ApJ, 478, 13

Odewahn, S.C. 1989, Ph.D. Thesis (Univ. of Texas)

Ostriker, J.P. \& Tremaine, S. 1975, ApJ, 202, L113

Press, W.H., Flannery, B.P., Teukolsky, S.A., \& Vetterling, W.T. 1992, Numerical Recipes, (Cambridge University Press: Cambridge)

Quinn, P.J., \& Goodman, J. 1986, ApJ, 309, 472
Scholz, R.-D., \& Irwin, M.J. 1994 in IAU Symp. 161, Wide Field Imaging, ed. H.T. MacGillivray

Tremaine, S.D. 1976, ApJ, 203, 72

Zaritsky, D. 1992, ApJ, 400, 74

Zaritsky, D. \& Rix, H.-W. 1997, ApJ, 478, 118

Zaritsky, D., Smith, R., Frenk, C., \& White, S.D.M. 1993, ApJ, 405, 464

Zaritsky, D., Smith, R., Frenk, C., \& White, S.D.M. 1997, ApJ, 478, L53 (ZSFW)

Zaritsky, D., \& White, S.D.M. 1994, ApJ435, 599 (ZW)

This 2-column preprint was prepared with the AAS LATEX macros v5.0. 


\section{Figure Caption}

Fig. 1. - A histogram of the satellite position angle relative to the disk major axis. The ZSFW sample (solid line) is compared to the predicted distribution (dashed line) from the Quinn \& Goodman (1986) model (adopting a limiting orbital inclination of $45^{\circ}$ and a satellite number density profile proportional to $r^{-1.8}$ ). Also shown is the angular distribution of ZSFW satellites beyond a projected radius of $200 \mathrm{kpc}$ (dotted line).

Fig. 2.- The azimuthal distribution of satellite galaxies. The upper panel displays the average position angle with respect to the disk for satellites in nearly equally populated radial bins (filled circles; left-hand axis) and the fraction of satellites to with position angles greater than $45^{\circ}$ in those same bins (open circles; right-hand axis). The bottom panel displays projected radius vs. position angle for the entire satellite sample. In both panels, the bold solid line represents the results from our simulations for Sample 1 and the best-fit $\theta_{l}$, $52^{\circ}$. 


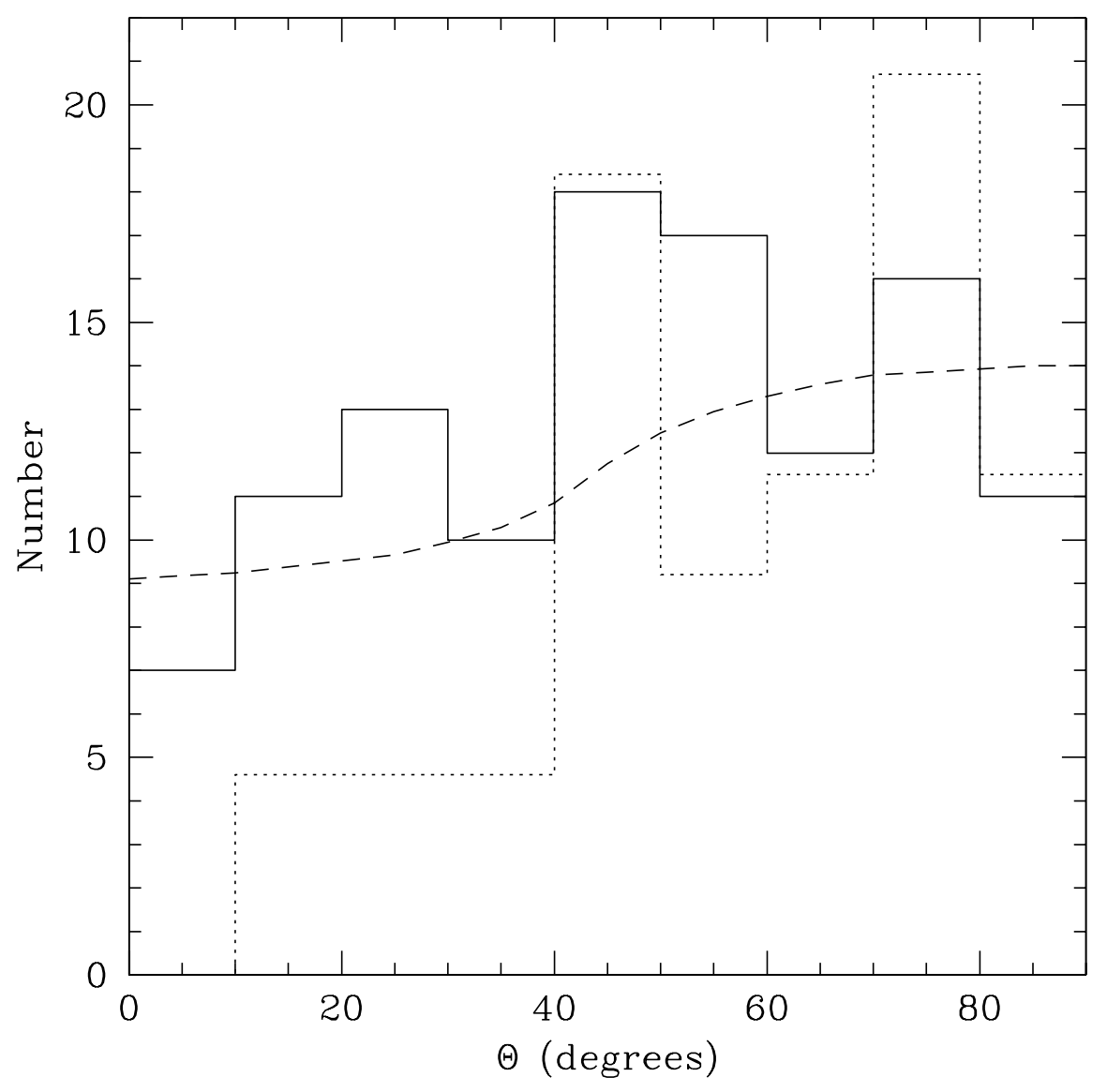




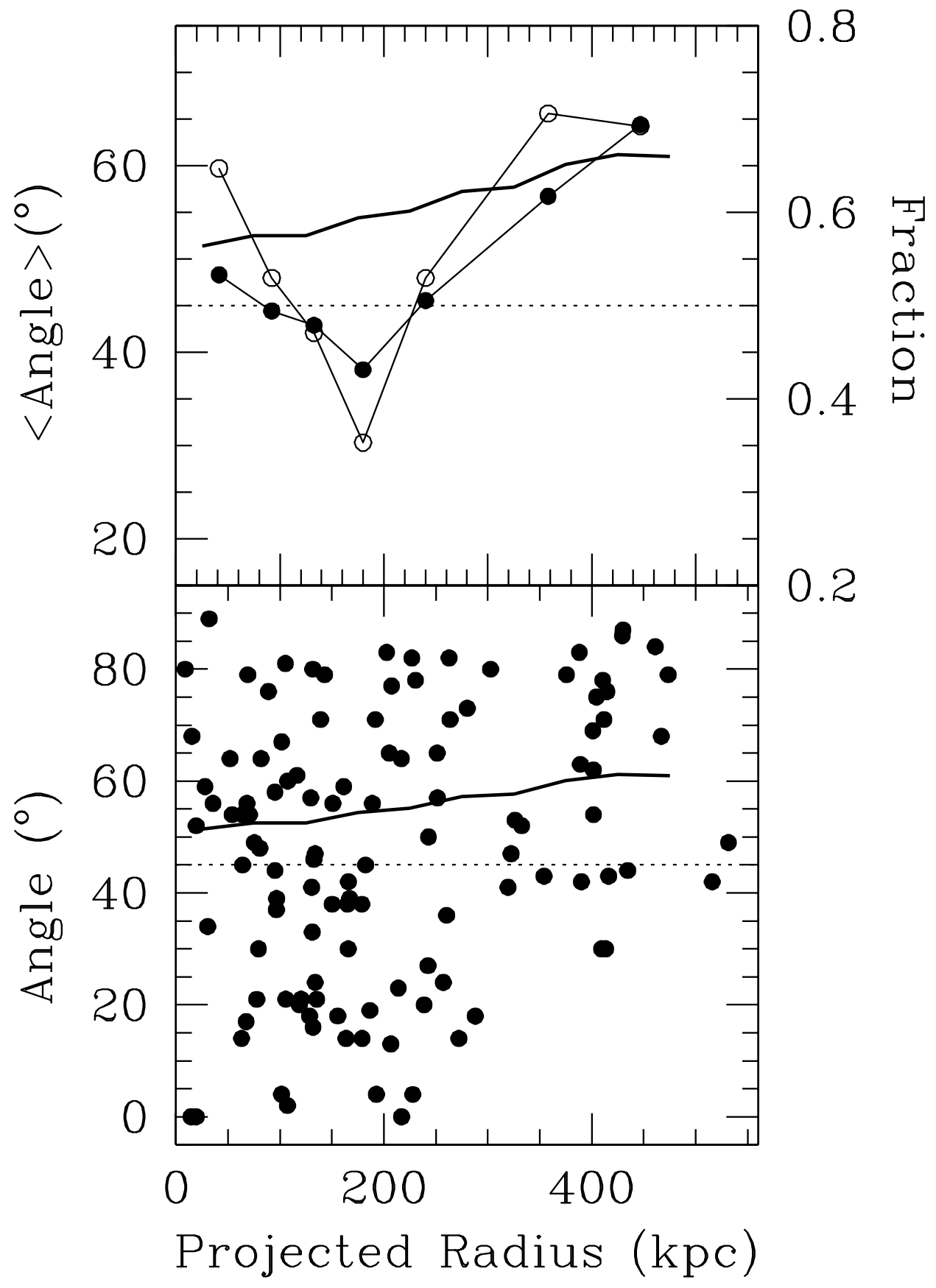


TABLE 1

Satellite Samples

\begin{tabular}{lcc}
\hline \hline Satellite Sample & Orbital Inclination Limit & Proj. Radius Limit \\
\hline 1 & none & none \\
2 & none & $r_{p}>300 \mathrm{kpc}$ \\
3 & $<45^{\circ}$ & none \\
4 & $<45^{\circ}$ & $r_{p}>200 \mathrm{kpc}$ \\
\hline
\end{tabular}

TABLE 2

Orbit Simulations

\begin{tabular}{lcccccc}
\hline \hline $\begin{array}{c}\text { No. } \\
(1)\end{array}$ & $\begin{array}{c}\text { Sample No. } \\
(2)\end{array}$ & $\begin{array}{c}\alpha \\
(3)\end{array}$ & $\begin{array}{c}f_{I N T} \\
(4)\end{array}$ & $\begin{array}{c}e \\
(5)\end{array}$ & $\begin{array}{c}\theta_{l} \text { (Kepler) } \\
(6)\end{array}$ & $\begin{array}{c}\theta_{l} \text { (Infall) } \\
(7)\end{array}$ \\
\hline 1 & 4 & 1.8 & 0.10 & 0.5 & $18^{\circ}[0,48]$ & $20^{\circ}[-,-]$ \\
2 & 4 & 1.8 & 0.10 & 0.7 & $18^{\circ}[0,58]$ & $12^{\circ}[-,-]$ \\
3 & 4 & 1.8 & 0.10 & 0.9 & $32^{\circ}[10,64]$ & $12^{\circ}[-,-]$ \\
4 & 4 & 1.5 & 0.10 & 0.5 & $18^{\circ}[0,52]$ & $\ldots$ \\
5 & 4 & 1.5 & 0.10 & 0.7 & $18^{\circ}[0,60]$ & $\ldots$ \\
6 & 4 & 1.5 & 0.10 & 0.9 & $30^{\circ}[6,66]$ & $\ldots$ \\
7 & 4 & 2.0 & 0.10 & 0.5 & $18^{\circ}[0,44]$ & $\ldots$ \\
8 & 4 & 2.0 & 0.10 & 0.7 & $18^{\circ}[0,58]$ & $\ldots$ \\
9 & 4 & 2.0 & 0.10 & 0.9 & $30^{\circ}[6,64]$ & $\ldots$ \\
10 & 4 & 1.8 & 0.05 & 0.5 & $16^{\circ}[0,50]$ & $\ldots$ \\
11 & 4 & 1.8 & 0.05 & 0.7 & $20^{\circ}[0,60]$ & $\ldots$ \\
12 & 4 & 1.8 & 0.05 & 0.9 & $32^{\circ}[10,64]$ & $\ldots$ \\
13 & 4 & 1.8 & 0.15 & 0.5 & $20^{\circ}[0,48]$ & $\ldots$ \\
14 & 4 & 1.8 & 0.15 & 0.7 & $18^{\circ}[0,58]$ & $\ldots$ \\
15 & 4 & 1.8 & 0.15 & 0.9 & $28^{\circ}[6,64]$ & $\ldots$ \\
16 & 3 & 1.8 & 0.10 & 0.5 & $48^{\circ}[2,90]$ & $36^{\circ}[0,90]$ \\
17 & 3 & 1.8 & 0.10 & 0.7 & $52^{\circ}[22,90]$ & $40^{\circ}[0,90]$ \\
18 & 3 & 1.8 & 0.10 & 0.9 & $68^{\circ}[34,90]$ & $40^{\circ}[0,90]$ \\
19 & 1 & 1.8 & 0.10 & 0.5 & $38^{\circ}[14,90]$ & $44^{\circ}[0,90]$ \\
20 & 1 & 1.8 & 0.10 & 0.7 & $52^{\circ}[24,90]$ & $40^{\circ}[0,90]$ \\
21 & 1 & 1.8 & 0.10 & 0.9 & $58^{\circ}[34,90]$ & $44^{\circ}[0,90]$ \\
22 & 2 & 1.8 & 0.10 & 0.5 & $12^{\circ}[0,44]$ & $12^{\circ}[-,-]$ \\
23 & 2 & 1.8 & 0.10 & 0.7 & $26^{\circ}[0,48]$ & $16^{\circ}[-,-]$ \\
24 & 2 & 1.8 & 0.10 & 0.9 & $34^{\circ}[16,54]$ & $16^{\circ}[-,-]$ \\
\hline
\end{tabular}

